\title{
Variability in Cognitive Aging: From Taxonomy to Theory
}

\author{
Ulman Lindenberger \\ Timo von Oertzen
}

Variability pervades cognitive aging. Shining examples of older individuals who preserved outstanding intellectual abilities well into very old age, such as Johann Wolfgang von Goethe or Sophocles, stand in contrast to individuals whose intellectual capacities are depleted by the time they reach later adulthood. Similar contrasts exist between different intellectual abilities. For example, if one looks at perceptual speed, one is likely to find monotonic decline after late adolescence and early adulthood. But if one looks at vocabulary, one will find age stability or positive change into very old age (Singer, Verhaeghen, Ghisletta, Lindenberger, \& Baltes, 2003).

Despite its prominence, the conceptual significance of variability in cognitive aging is difficult to evaluate. On the one hand, variability in old age is often portrayed as the late-life culmination of interacting developmental causes that affect different individuals and different task domains to varying degrees. Here, variability in intellectual functioning is seen as a dependent variable, or outcome (for an example, see Figure 7 in Lindenberger \& Baltes, 1997). On the other hand, variability can also be conceived as an agent, or mechanism, of senescent changes in cognition (Hultsch \& MacDonald, 2004; Li, Lindenberger, \& Sikström, 2001; Thaler, 2002). According to this complementary position, certain forms of variability index basic properties of senescing cognitive systems at neural or behavioral levels of analysis, and may help to explain ontogenetic changes in cognition from early adulthood to old age.

Thus, the conceptual status of variability is ambiguous, as it denotes both consequences and causes of development. The same kind of ambiguity holds for other central phenomena of cognitive aging. For instance, the ubiquitous slowing of information processing with advancing age is conceived both as an antecedent (e.g., Salthouse, 1996) and as a consequence (e.g., Ratcliff, Spieler, \& McKoon, 2000) of other senescent changes in behavior.

The general objective of the present chapter is to help in categorizing and clarifying the conceptual sta- 
tus of variability in cognitive aging. We first introduce a taxonomy of intra-individual variability. Then, we evaluate the common practice of measuring variability between persons to test propositions about withinperson variability. Third, we focus on two variability phenomena in cognitive aging: high processing fluctuation (i.e., low processing robustness), and intellectual ability dedifferentiation. Fourth and finally, we report a neurocomputational model that integrates evidence about variability in cognitive aging across neural and behavioral levels of analysis and across microgenetic and ontogenetic timescales. We show how this neurocomputational model links ability dedifferentiation to low processing robustness, and may further guide the investigation of variability in cognitive aging

\section{A TAXONOMY OF WITHIN-PERSON VARIABILITY}

Following Cattell's (1966) data box of individuals, variables, and occasions, Buss (1974) presented a schema of inter-individual differences and intra-individual changes that constituted 15 different data-gathering strategies for studying variability. Recently, S.-C. Li, Huxhold, and Schmiedek (2004) modified this picture by focusing on intra-individual variability and by adding substantive assumptions about its timescale and scope. A modified version of this scheme is presented in Table 21.1.

The taxonomy shown in Table 21.1 coordinates two dichotomous dimensions, timescale and scope. On the timescale dimension, we follow the distinction between microgenetic variations and ontogenetic change (e.g., Lindenberger \& Baltes, 1995; Siegler, 1989). Microgenetic variations are short term and often reversible, whereas ontogenetic changes are long term and often cumulative, progressive, and permanent. Both microgenetic and ontogenetic changes can be adaptive, maladaptive, or both (i.e., adaptive with respect to one developmental goal but maladaptive with respect to another; cf. Baltes, 1987). On the scope dimension, alterations in a single function are set apart from transformations in functional organization involving more than one function. Alterations in a single function are often assumed to be local in characterthat is, confined to the function under study. However, both microgenetic variations and ontogenetic change may co-involve more than one function and entail global reorganization (structural modification). Such system-general transformations necessitate a multivariate approach.

A further dimension not explicitly represented in Table 21.1 is the distinction between neural and behavioral levels of analysis. Most forms of within-person variability can be studied at both neural and behavioral levels. For instance, age-associated decline in episodic memory performance during adulthood and old age can be studied from a neural perspectivewhat are its anatomical, neurochemical, and neurofunctional correlates? - but it also can be studied from a behavioral perspective: what are its behavioral antecedents, correlates, and consequences?

For illustration, we may apply this classificatory scheme to one specific cognitive function such as episodic memory. At the microgenetic timescale, trialto-trial variability in the ability to represent contextual information at encoding would qualify as univariate microgenetic variability (i.e., the upper left cell of Tabie 21.1). Shifts in resource allocation between an episodic memory task and a simultaneously performed perceptual-motor task are instances of functional reorganization (upper right cell). At the ontogenetic timescale, longitudinal age changes in episodic memory ability from young adulthood to old age, when viewed in isolation, fall into the univariate, local category (lower left cell). In contrast, ontogenetic changes in the functional relation between episodic and semantic memory represent an instance of global functional reorganization (lower right cell).

In our view, a major challenge for cognitive aging research is to identify neural and behavioral mechanisms that link local to global variations and microgenetic variations to ontogenetic change. Specifically, developmental researchers need to specify how microgenetic variations are affected by and result in ontogenetic change, and how local variability is affected by and results in global organization, both neurally and behaviorally. In other words, if we wish to move from taxonomy to theory, we need to explicate mechanisms that link developmental phenomena across the cells of our classificatory scheme. Based on the criteria of parsimony and explanatory power, mechanisms that link local microgenetic variations to global ontogenetic transformations seem especially attractive. One such hypothetical mechanism, the stochastic gain parameter of connectionist networks, will be described in the last section of this chapter. 
TABLE 21.1. Taxonomy of within-person variability in cognitive functioning across the lifespan

\begin{tabular}{|c|c|c|}
\hline \multirow[b]{2}{*}{ Time scale } & \multicolumn{2}{|c|}{ Scope } \\
\hline & $\begin{array}{l}\text { Variations in a Single Function } \\
\text { (e.g., local, univariate) }\end{array}$ & $\begin{array}{l}\text { Transformations in Functional Organization (e.g., } \\
\text { global, multivariate) }\end{array}$ \\
\hline $\begin{array}{l}\text { Microgenetic } \\
\text { (e.g., usually } \\
\text { across trials, } \\
\text { sessions, or } \\
\text { weeks) }\end{array}$ & $\begin{array}{l}\text { Relatively reversible variations in one function } \\
\text { Examples: } \\
\text { - processing fluctuation (processing liability } \\
\text { or lack of processing robustness) } \\
\text { - neural and behavioral plasticity (short-term } \\
\text { learning potential) } \\
\text { - within-task strategic diversity (richness of } \\
\text { within-task behavioral repertoire) } \\
\text { - adaptability/resilience to environmental } \\
\text { perturbations } \\
\text { - cyclic (e.g., state) variations in any specific } \\
\text { function }\end{array}$ & $\begin{array}{l}\text { Relatively reversible variations in functional } \\
\text { organization } \\
\text { Examples: } \\
\text { - shifts in resource allocation, coordination and } \\
\text { compensatory behavior during multitasking } \\
\text { - context-driven variations in mental set and } \\
\text { functional organization (e.g., posture control } \\
\text { with eyes open or closed) } \\
\text { - situational choice and preference behavior }\end{array}$ \\
\hline $\begin{array}{l}\text { Ontogenetic } \\
\text { (e.g., usually } \\
\text { across } \\
\text { months, } \\
\text { years, or } \\
\text { decades) }\end{array}$ & $\begin{array}{l}\text { Relatively permanent (e.g., cumulative, } \\
\text { progressive) changes in one function } \\
\text { Examples: } \\
\text { - physical growth } \\
\text { - progressive (e.g., trait) changes in any } \\
\text { specific cognitive function (e.g., mechanics } \\
\text { of cognition / broad Gf, episodic memory) } \\
\text { - long-term learning and skill acquisition } \\
\text { (e.g., pragmatics of cognition / broad Gc, } \\
\text { semantic memory) }\end{array}$ & $\begin{array}{l}\text { Relatively permanent (e.g., cumulative, progressive) } \\
\text { alterations in functional organization } \\
\text { Examples: } \\
\text { - ability dedifferentiation from adulthood to old age } \\
\text { - ability differentiation from childhood to early } \\
\text { adulthood } \\
\text { - corticogenesis and functional specification of } \\
\text { brain areas during maturation and learning } \\
\text { - functional reintegration of brain circuitry in } \\
\text { old age } \\
\text { - shrinkage of brain volume and loss of } \\
\text { receptor density during senescence } \\
\text { - cortical reorganization after brain damage } \\
\text { or trauma }\end{array}$ \\
\hline
\end{tabular}

Note: This taxonomy is not meant to be exhaustive. For instance, societal sources of variability are not systematically considered. All listed forms of variability can be studied at neuronal and behavioral levels of analysis. Examples are drawn from both levels. Examples printed in italics are discussed in more detail in this chapter. A major challenge for lifespan psychology is to identify mechanisms that link local to global variations, microgenetic variations to ontogenetic change, and neuronal mechanisms to behavior. Theories that link neuronal mechanisms in a single function acting at a microgenetic timescale to global ontogenetic transformations in behavior are high in parsimony and explanatory power. (Modified after S.-C. Li, Huxhold, \& Schmiedek, 2004.)

\section{MEASURING VARIABILITY WITHIN AND BETWEEN INDIVIDUALS}

Whereas developmental theories about variability generally refer to ontogenetic changes and microgenetic variations within individuals, most of the data used to evaluate such theories refer to variability between individuals. This habitual mismatch between theory and data has been noted for a long time in lifespan psychology (e.g., Baltes, Reese, \& Nesselroade, 1977/1988), child development (e.g., Reuchlin, 1978), dynamic systems approaches (e.g., Smith \& Thelen, 2003), and proponents of an idiographic approach (e.g., Magnusson \& Stattin, 1998).

Though many researchers are aware of the mismatch, its consequences are often overlooked or belittled. The study of univariate ontogenetic changes forms a notable positive exception to this rule. Here, the difference between intra- and inter-individual variation generally refers to the distinction between cross-sectional age differences and longitudinal age changes. The benefits and costs of both data-analytic schemes, and the issue of extrapolating average age changes on the basis of cross-sectional age differences 
and observed longitudinal changes, are fairly well understood (e.g., Baltes, 1968; Lindenberger \& Baltes, 1994; Lövdén, Ghisletta, \& Lindenberger, 2004; Salthouse, 1991; Schaie, Maitland, Willis, \& Intieri, 1998). For instance, cross-sectional age differences are confounded with stable differences between birth cohorts, whereas longitudinal observations contain retest effects and are often conditioned by selective attrition (e.g., Lindenberger, Singer, \& Baltes, 2002).

In contrast, much less is known about the relationship between intra- and inter-individual variability with respect to global ontogenetic changes in functional organization, univariate microgenetic variations, and multivariate microgenetic variations (i.e., the remaining three cells of the cross-classification of timescale and scope of variability presented in Table 21.1). Cognitive aging researchers often ignore the relation between intra-individual and inter-individual variability, and simply substitute intra-individual with inter-individual observations, primarily because the latter are more easily available than the former. The methodological assumptions underlying this strategy are discussed in the next section.

\section{Extrapolating Intra-Individual Variability Based on Inter-Individual Variability}

The generalizability of inter-individual to intra-individual variability hinges on two interrelated criteria: variation equivalence and sample homogeneity (Lövdén \& Lindenberger, 2005). We use the term variation equivalence to designate an empirical situation in which intra-individual and inter-individual variability do not differ in important ways with respect to the variables under study. Variation equivalence holds when the processes that generate variability between and within individuals are identical, but it may also hold for other reasons (e.g., by coincidence). Formal criteria that need to be met to guarantee variation equivalence depend on statistical context (e.g., Markov chains, Bayesian nets) and have been discussed under the heading of ergodicity (e.g., Molenaar, Huizenga, \& Nesselroade, 2003). A formal treatment of these criteria is beyond the scope of the present chapter.

Equivalance of inter-individual and intra-individual variability is often assumed but rarely tested. Inagine, for instance, a correlation of $r=.50$ between episodic memory and semantic memory that was ob- tained by administering indicators of the two abilities to different individuals at a given point in time. In the absence of intra-individual information, such a between-person correlation is often meant to suggest a moderately positive correlation within individuals, perhaps reflecting the existence of two separate but overlapping memory systems. One way to test this interpretation empirically would actually be to obtain extensive observations on measures of episodic and semantic memory within individuals. Even for the same sample of individuals, the correlation based on between-person variability does not have to approximate the mean of the distribution of the withinperson correlations, nor does it prescribe its range. For instance, in the case of an $r=.50$ between-person correlation between episodic and semantic memory, within-person correlations may be $r=-.30$ in some individuals and $r=.80$ in others.

Sample homogeneity refers to the assumption that structural relations among variables of interest do not differ significantly from person to person. In the present case, sample homogeneity requires that the correlations between episodic and semantic memory be of similar magnitude within all individuals (e.g., a sufficiently narrow nomal distribution around the same mean). Sample homogeneity is a necessary but not a sufficient condition for variation equivalence. In our previous example, the intra-individual correlations between episodic and semantic memory may be homogeneously distributed around $r=.30$, but the inter-individual correlation may still be $r=.50$.

The assumption of sample homogeneity is at odds with the more general notion that individuals differ in the way they change over time. According to lifespan theory (e.g., Baltes et al., 1977/1988; cf. Lindenberger \& Baltes, 1999; Tetens, 1777), the developmental path of each individual corresponds to a multivariate pattern of intra-individual change. Description and explanation of these intra-person patterns is a prerequisite for arriving at unbiased descriptions and explanations of the differences and commonalities among them. Premature aggregation across individuals can mask both differences and commonalities in change (e.g., Estes, 1956; Wohlwill, 1973). In the extreme case, certain laws and regularities may be specific to aggregate data and not apply to any of the individuals on which the aggregate data were based.

To conclude, the quid pro quo approach to variability, in which inter-individual variability is taken 
as a valid proxy for intra-individual variability, mandates equivalence between inter-individual and intraindividual variation, and presupposes homogeneity of change. Except for univariate ontogenetic changes we know exceedingly little about the degree to which these assumptions are met.

\section{Illustrating the Difference Between Inter- and Intra-Individual Variability}

The following illustration further specifies the difference between inter-individual and intra-individual variability. It uses the metaphor of leaves falling down to earth (see Figure 21.1). The movements of any given leaf falling down to earth will differ from the movements of all other leaves as a function of shape, weight, air pressure, initial conditions, and so on. Plyysicists may want to arrive at general laws of leaf falling that generalize across all possible constellations of variables (e.g., apply to all possible leaves). Such general laws would accommodate both the differences and commonalities in movement among various types of leaves. Probably, gravity and turbulence will play prominent roles in formulating such laws. For instance, all leaves eventually reach the ground because of gravity but each leaf moves down to earth in a slightly different manner because of turbulence.

To arrive at such laws, physicists probably would refrain, except for special cases, from using averages over different leaves as their primary database. In fact, the trajectory of the "average leaf" would be more similar to a stone falling down to earth in slow motion than to the falling patterns of any existing leaf because movements due to turbulence would cancel each

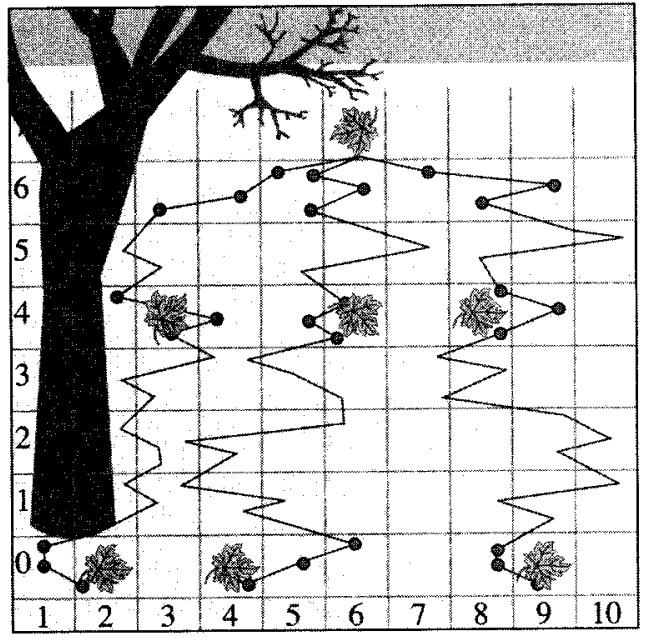

FIGURE 21.1. Illustrating the difference between intraindividual and inter-individual variability. The picture shows the path of three leaves falling from a tree to the ground. The dots correspond to observations, and are summarized in Table 21.2.

other out. Thus, the movements of the average leaf would not provide a good empirical basis for arriving at adequate models of leaf falling.

Figure 21.1 depicts the positions of three individual leaves on their way down to earth. The three leaves start falling at a vertical position (i.e. height) of 8 meters and a horizontal position of 6 meters, and their horizontal positions are repeatedly measured as a function of height as they are falling down to earth. Table 21.2 reports the means and variances in hori-

TABLE 21.2. Means and standard deviations in horizontal position of three individual leaves and the "average leaf": Illustrating the difference between intra-individual and inter-individual variability

\begin{tabular}{|c|c|c|c|c|c|c|c|c|c|c|c|c|}
\hline \multirow[b]{3}{*}{$\begin{array}{l}\text { Vertical Position } \\
\text { (range in meters) }\end{array}$} & \multicolumn{12}{|c|}{ Horizontal Position (meters) } \\
\hline & \multicolumn{3}{|c|}{ left leaf } & \multicolumn{3}{|c|}{ middle leaf } & \multicolumn{3}{|c|}{ right leaf } & \multicolumn{3}{|c|}{ all leaves } \\
\hline & scores & mean & SD & scores & mean & $S D$ & scores & mean & SD & mean & SD & $\begin{array}{l}\text { mean of } \\
\text { intra-SDs }\end{array}$ \\
\hline $6-7$ & $5,4,3$ & 4.00 & 0.82 & $5,6,5$ & 5.33 & 0.47 & $7,8,8$ & 7.67 & 0.47 & 5.67 & 1.81 & 0.59 \\
\hline $4-5$ & $2,4,3$ & 3.00 & 0.82 & $6,5,6$ & 5.67 & 0.47 & $8,9,8$ & 8.67 & 0.47 & 5.67 & 2.26 & 0.59 \\
\hline $0-1$ & $1,1,2$ & 1.33 & 0.47 & $6,5,4$ & 5.00 & 0.82 & $8,8,9$ & 8.33 & 0.47 & 4.89 & 2.92 & 0.59 \\
\hline
\end{tabular}

Note: Date are illustrated in Figure 21.2. 
7.ontal position for these leaves $6-7,4-5$, and $0-1$ meters above the ground. Intra-individual standard deviations indicate that the leaves' intra-individual variability in movement stays relatively constant over time; in fact, the average intra-individual standard deviation (i.e., mean of SDs) stays constant. In contrast, inter-individual standard deviations (i.e., the overall SD) increase over time, reflecting the fact that the three leaves diverge in horizontal position over time. The corresponding comparisons of central tendency yield opposite results. Here, the intra-individual means show greater divergence over time than the inter-individual average (i.e., the overall mean).

This illustration demonstrates that empirical results based on inter-individual variability may not always be appropriate to test propositions about intra-person mechanisms. It also suggests that endorsing individuals as privileged units of analysis does not mandate a withdrawal from the search for laws of behavioral development that generalize across collections of individuals. Instead, chances of arriving at such laws may increase, rather than decrease, when intra-individual variability is brought to the fore.

\section{FMPIRICAL FINDINGS ABOUT VARIABILITY IN COGNITIVE AGING}

I'he empirical investigation of variability in cognitive aging has intensified in recent years. In the following, we concentrate our discussion on two central phenomena: processing fluctuation and ability dedifferentiation. Whereas processing fluctuation (i.e., processing lability or lack of processing robustness) operates at the microgenetic timescale, ability dedifferentiation is operating at an ontogenetic timescale (see also Table 21.1). After a selective review of empirical findings, we report a neurocomputational model, originally introduced by S.-C. I,i and Lindenberger (1999), that links ability dedifferentiation to lack of processing robustness by means of a hypothetical neuronal mechanism.

\section{Processing Fluctuation}

Processing fluctuation, or lack of processing robustness, refers to a predominantly maladaptive form of microgenetic variability. For instance, in the context of a choice-reaction time task, processing fluctuation would correspond behaviorally to response time variability within a given experimental condition. Here, greater variability indicates that individuals are less able to reproduce their behavior over time. Little is known about adult age changes in processing fluctuation, and about the relative magnitude of such fluctuations in comparison to ontogenetic age trends in mean levels of functioning. Based on the general notion that senescence produces an increasingly labile, less efficiently regulated internal milieu (T'haler, 2002), and for more specific reasons outlined in the next section, we expect that processing fluctuation is increasing with advancing adult age.

Nesselroade and Salthouse (2004) investigated cross-sectional adult age differences in processing fluctuation in adults ranging from 20 to 91 years of age. Research participants were asked to perform three perceptual-motor tasks on three different occasions administered within a two-week period. For each task, three types of variability (i.e., standard deviations) were computed: (1) standard between-person variability; (2) within-person, within-session variability, computed as a person's average within-session standard deviation over three repeated administrations of each task; (3) within-person, between-session variability, computed as the standard deviation of each person's mean session scores.

Nesselroade and Salthouse (2004) made several important observations. First, the two indices of processing fluctuation showed substantial positive correlations within and across tasks, indicating that people differed reliably and consistently in variability of perceptualmotor performance. Second, when computing ratios of within-person over between-person variability, Nesselroade and Salthouse (2004) discovered that the magnitude of both types of within-person variability amounted to about half the magnitude of the standard measures of inter-individual variability. 'The corresponding ratios ranged from 0.31 to 0.85 , with the majority being in the $0.40-0.55$ range. In fact, the average fluctuation of a single individual within a single session, if projected onto average age trends, corresponded to an age range of 21-44 years! In other words, depending upon whether a given 32-year-old individual performed more or less variably (e.g., "had a good or a bad day"), he or she would look like an average 21year-old or an average 44-year-old individual. Third, intra-individual fluctuation was negatively related to performance level, confirming its dysfunctional character. Fourth, intra-individual fluctuations increased with age, supporting the hypothesis that the cognitive system functions less reliably with advancing age. 
The consistency, magnitude, external validity, and age association of within-person variability observed by Nesselroade and Salthouse (2004) have profound implications for cognitive aging research. Methodologically, they demonstrate that measures of central tendency fail to represent the performance characteristics of a given individual. A major portion of variability commonly attributed to between-person differences or measurement error appears to be due to within-person fluctuations. As noted by Nesselroade and Salthouse (2004), these findings challenge "the value of even a 'working' notion of the classical test theory conception of true score"(p. 53). As a consequence, attempts to resolve methodological problems associated with developmental sample heterogeneity by relying on so-called narrow age-cohort designs; that is, the comparative study of strictly age-homogeneous samples (Hofer \& Sliwinski, 2001) do not appear to be particularly promising. If within-person microgenetic variability corresponds to several decades of average ontogenetic age gradients, and if within-person variability increases with age, then matching individuals with respect to chronological age does not effectively control for developmental status.

Instead, it seems more promising to incorporate intensive within-person observations, or measurement bursts (Nesselroade, 1991), into the design of longitudinal studies to explicitly study interactions among microgenetic and ontogenetic forms of variability such as processing fluctuation, plasticity (short-term learning potential), and long-term development (cf. Lindenberger \& Baltes, 1995; Siegler, Chapter 20, this volume; for recent empirical examples, see MacDonald, Hultsch, \& Dixon, 2003; Singer, Lindenberger, \& Baltes, 2003). Given that empirical associations between perceptual-motor performance of the kind measured by Nesselroade and Salthouse (2004) and other aspects of intellectual and sensorimotor performance are substantial, especially in aging samples (Baltes \& Lindenberger, 1997; Li \& Lindenberger, 2002; Lindenberger \& Baltes, 1997), this conclusion is likely to generalize to many behavioral domains.

Of course, not all forms of microgenetic variability are maladaptive. Contradictory findings about the functional meaning of microgenetic variability can be reconciled by separating mechanisms supporting skill acquisition and resilience to environmental perturbations from mechanisms permitting stable performance at asymptotic levels of learning. According to this view, a variety of mechanisms contribute to observed microgenetic variability. For instance, during early phases of learning and with difficult reasoning tasks, microgenetic variability may be dominated by strategic diversity - that is, by an individual's capacity to approach the task in many different ways (Chapter 20, this volume). The contribution of strategic diversity to variability may fade out late in learning when optimal behavioral repertoires have been selected, trained, and automatized. Furthermore, its contribution may be small from the very start whenever tasks are sufficiently simple or constrained to effectively prevent strategic diversity. Processing fluctuation is likely to be present throughout learning, but its relative contribution to overall microgenetic variability may increase as the contribution of strategic diversity decreases. In addition, processing fluctuation may also increase in absolute magnitude with learning because the production of asymptotic performance may tax the system's capacity limits, thereby rendering its behavior more susceptible to minor external and internal perturbations.

Based on these considerations, we expect that interindividual differences in within-person variability early in learning should be positively related to interindividual differences in performance level, reflecting differences in strategic diversity. In contrast, variability late in learning should be negatively related to performance level, primarily reflecting differences in processing fluctuations. Thus, the association between variability and performance should switch signs in the course of learning. The results of a recent study (Allaire $\&$ Marsiske, 2002) exactly followed this pattern.

In sum, processing fluctuation, a maladaptive form of microgenetic variability, can be conceptually and empirically dissociated from other, more beneficial forms of microgenetic variability such as strategic diversity and short-term learning. Moreover, recent evidence (Nesselroade \& Salthouse, 2004) suggests that processing fluctuation in perceptual-motor cognitive tasks is of considerable magnitude in relation to ontogenetic age trends in mean levels of performance, and increases significantly from adulthood to old age.

\section{Ability Dedifferentiation in Old Age}

Within the psychometric research tradition, the differentiation/dedifferentiation hypothesis is arguably the most comprehensive proposition about lifespan changes in variability (cf. Lindenberger, 2001). The 
hypothesis asserts that the functional organization of intellectual abilities is relatively compressed in childhood, unfolds (differentiates) during maturation (e.g., Garrett, 1946), and contracts (dedifferentiates) again in old age (e.g., Baltes, Cornelius, Spiro, Nesselroade, $\&$ Willis, 1980; Reinert, 1970). During childhood and old age, operation and expression of intellectual abilities are assumed to depend strongly on system-general constraints, reflecting the age-graded developmental status of relevant biological substrates. With maturation and during adulthood, these system-general ensembles of constraints are relaxed, and other factors, such as interest, motivation, and occupational/educational opportunities, determine intellectual development to a relatively greater degree, leading to greater diversity in levels of functioning across different intellectual abilities. As such, the hypothesis conveys a dynamic view of the structure of intellectual abilities (cf. Krampe \& Baltes, 2003). The hypothesis is specified further by two-component theories of intellectual development that distinguish between biological and cultural dimensions of cognition (Baltes, 1987; Cattell, 1971; Horn, 1989; Tetens, 1777; for comparison, see Baltes, Lindenberger, \& Staudinger, 1998; Lindenberger, 2001). The general constraints assumed to operate more strongly early and late in ontogeny are assumed to be biological in kind.

Clearly, the differentiation/dedifferentiation hypothesis of lifespan intelligence refers to the organization of intellectual abilities within individuals. However, almost all of the empirical evidence brought to bear on this hypothesis consists in comparing the structure of inter-individual differences (i.e., betweenperson variability) in different age groups, such as decreasing correlations among intellectual tests sampled across persons from childhood to adolescence, and increasing correlations among intellectual tests sampled across persons from adulthood to old age. Thus, just as described in the previous section, evidence obtained by structuring variability between individuals has been used routinely to test a hypothesis that refers to age changes in functional organization within individuals.

Most of the evidence based on inter-individual differences seems to favor the existence of ability dedifferentiation in advanced old age (e.g., Baltes \& Lindenberger, 1997; Hultsch, Hertzog, Dixon, \& Small, 1998;Li, Lindenberger, et al., 2004; Schaie et al., 1998; but see Park et al., 2002). Discrepancies in findings may relate to sample composition, differ- ences in age groups, and restriction of range (e.g., Deary et al., 1996; Nesselroade \& Thompson, 1995). Only a few studies have examined the dedifferentiation hypothesis at the intra-person level, by assessing age differences in ability covariance structures within young and older adults (for a review on the relation between sensorimotor and intellectual domains, see Li \& Lindenberger, 2002). Here, the evidence generally appears to be supportive as well (e.g., Li, Aggen, Nesselroade, \& Baltes, 2001).

\section{Links Between Dedifferentiation and Processing Fluctuation}

Recently, S.-C. Li, Lindenberger, et al. (2004) empirically linked inter-individual differences in microgenetic processing fluctuation to the differentiation/ dedifferentiation hypothesis of lifespan intelligence. The authors administered a psychometric battery comprising 15 tests assessing three marker abilities of fluid mechanics (perceptual speed, reasoning, and fluency) and two marker abilities of crystallized pragmatics (verbal knowledge and fluency) to a sample of 291 individuals ages 6-89 years. Participants were classified into six age groups, childhood (6-11 years), adolescence ( $12-17$ years), early adulthood (18-35 years), middle adulthood ( $26-54$ years), late adulthood ( 55 69 years), and old age ( $70-89$ years). In addition, $\mathrm{Li}$, Lindenberger, et al. (2004) also administered five basic experimental cognitive tasks (i.e., visual search, response competition, memory search, and choice reactions). Based on performance on these tasks, the authors computed two overall indicators of information processing: processing speed (i.e., a person's average speed of responding across the five tasks) and processing robustness (i.e., the inverse of a person's average within-task reaction-time fluctuation)

Two sets of findings are relevant in the present context (see Figure 21.2). First, within the limitations of the inter-individual variability approach, results provided support for the differentiation/dedifferentiation hypothesis. Across the lifespan, fluid intelligence, crystallized intelligence, processing speed, and processing robustness all followed the expected inverted U-shape pattern. Fluid intelligence showed the earliest increase during early and the earliest decrease in late life, whereas crystallized intelligence showed a later increase and a later decrease. Principal component analyses of the 15 psychometric tests were performed separately in each group. In childhood, late 

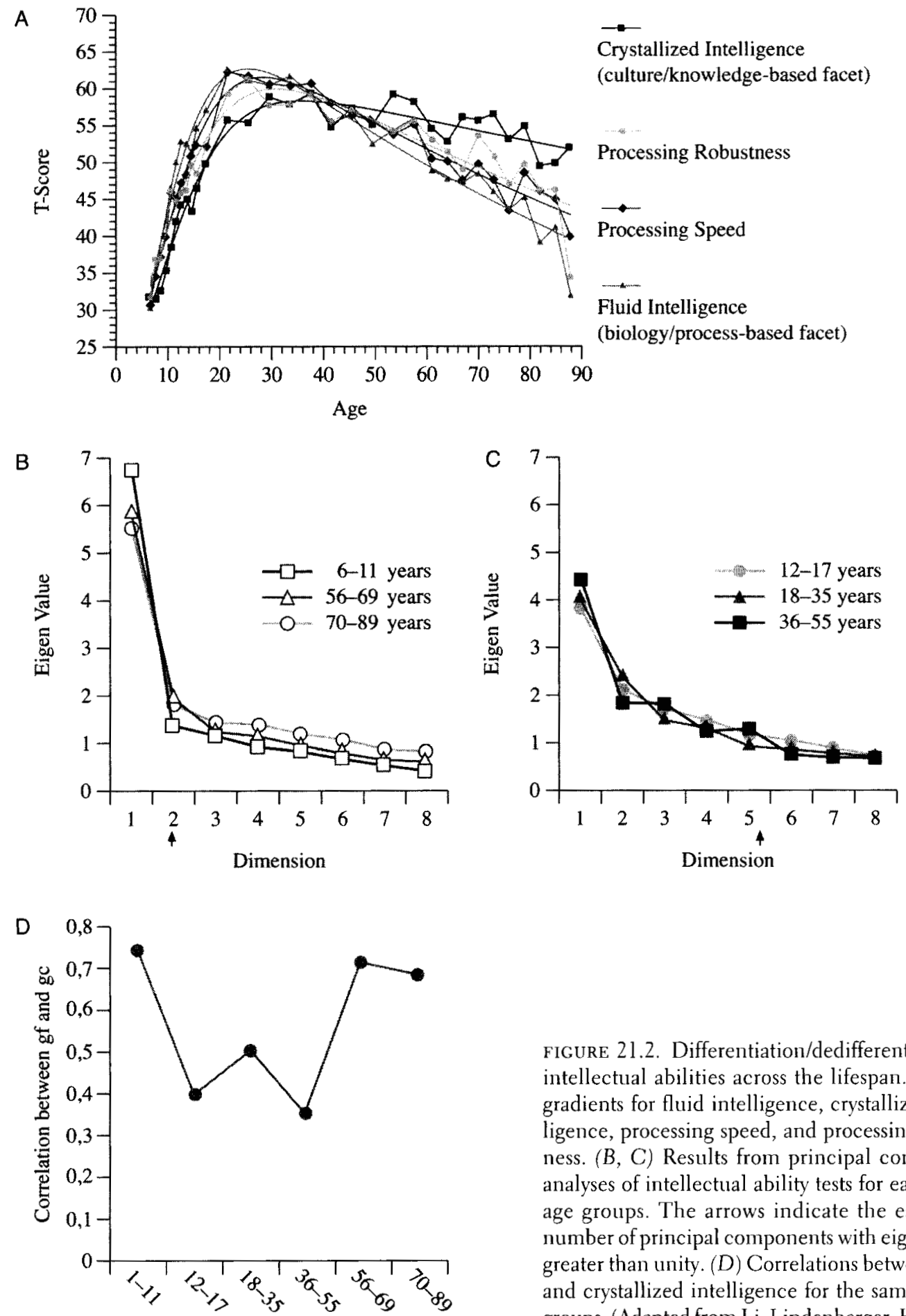

Age Group

FIGURE 21.2. Differentiation/dedifferentiation of intellectual abilities across the lifespan. (A) Age gradients for fluid intelligence, crystallized intelligence, processing speed, and processing robustness. ( $B, C$ ) Results from principal component analyses of intellectual ability tests for each of six age groups. The arrows indicate the estimated number of principal components with eigenvalues greater than unity. (D) Correlations between fluid and crystallized intelligence for the same six age groups. (Adapted from Li, Lindenberger, Hommel, Aschersleben, Prinz, \& Baltes, 2004.) 
adulthood, and old age, only two components with eigenvalues greater than 1 were extracted. In adolescence, young, and middle adulthood, five components displayed eigenvalues greater than unity. Also, fluid and crystallized intelligence were more highly correlated in childhood, late adulthood, and old age than in adolescence, young, and middle adulthood.

Second, processing speed was a strong predictor of intellectual performance in all age groups, and especially in childhood and old age. In contrast, the unique predictive validity of processing robustness was restricted to late adulthood and old age (see Table 21.3). Thus, in late adulthood and old age, higher processing fluctuation was uniquely associated with lower performance on psychometric measures of intelligence. This empirical association between high processing fluctuation, low average performance, and compressed ability structure will be taken up again in the next section.

\section{Dedifferentiation at the Neuronal Level}

The behavioral neuroscience of aging has been remarkably successful in promoting variability as a key concept in research on aging. Regional covariation methods suggest that functional interconnections found in younger adults are typically weaker in older adults (e.g., Schreurs, Bahro, Molchan, Sunderland, $\&$ Mclntosh, 2001). With respect to activated brain areas, both under-and overactivation relative to young adults have been observed. Possibly, some of the observed age changes in activation patterns reflect a loss of distinctiveness (or dedifferentiation) of mental representations (Park, Polk, Park, Minear, Savage, \& Smith, 2004). In addition, some increments in the bilaterality of prefrontal activation may represent adaptive (compensatory) changes in functional circuitry (Cabeza, 2002).

Recently, strong evidence in favor of dedifferentiation at the intra-person, neuronal level of analysis has been found (Park et al., 2004). Park et al. examined whether neural structures become less functionally differentiated and specialized with age, as predicted by the differentiation/dedifferentiation hypothesis. Whereas much of the earlier work has focused on frontal cortex, this study investigated age differences in specificity of regions in ventral visual cortex. Compared to frontal areas, ventral visual cortex shows considerably less neurodegenerative change (Raz, 2000). Also, in young adults, this area is known to respond
TABLE 21.3. Commonality analyses of processing robustness and processing speed as predictors of fluid and crystallized intelligence in late adulthood and old age (55-89 years)

\begin{tabular}{lcc}
\hline & \multicolumn{2}{c}{ Dependent variables } \\
\cline { 2 - 3 } Predictors & $\begin{array}{c}\text { Fluid } \\
\text { Intelligence }\end{array}$ & $\begin{array}{c}\text { Crystallized } \\
\text { Intelligence }\end{array}$ \\
\hline Unique processing robustness & 4.7 & 0.1 \\
Unique processing speed & 4.1 & 4.4 \\
Shared & 28.7 & 13.3 \\
Total & 37.5 & 13.5 \\
\hline
\end{tabular}

Note: Components different from zero are repinted in boldface $(p<.01)$. (Adapted from Li, Lindenberger, Hommel, Aschersleben, Prinz, \& Baltes, 2004.)

differentially to different visual categories. Park et al. (2004) administered stimuli from four different visual categories-faces, houses, pseudowords (plausible non-words), and chairs - to young and old adults, and assessed neural activity during stimulus perception using functional magnetic resonance imaging. Within the ventral visual cortex, the authors isolated, separately for each participant, the most active regions for each of the stimulus categories relative to phasescrambled control stimuli. Note that this person-based procedure takes care of inter-individual differences in brain activation patterns, thereby avoiding some of the problems commonly associated with averaging brain activity across individuals.

Young adults showed clear peaks in activation profiles. For instance, when processing faces, the voxels selected because of their activation peak during face perception were much more active than the voxels selected because of activation peaks for houses, pseudowords, or chairs. In contrast, old adults showed considerably flatter performance profiles, though the overall amount of activation relative to control stimuli was about the same. For instance, when processing faces, the activation differences between voxels selected because of their activation peak for faces and voxels selected because of corresponding activation peaks for the other three categories were small. These findings demonstrate that visual processing in the neocortex becomes less functionally specialized with advancing age, perhaps reducing the distinctiveness of cortical representations (cf.Li \& Sikström, 2002). 


\section{A FORMAL MODEL OF VARIABILITY IN COGNITIVE AGING}

Formalization of theoretical assumptions is a particularly powerful tool in science. When applied to cognitive aging, such models enable researchers to map differences observed at neural and behavioral levels of analysis onto model parameters that express theoretical assumptions about the ontogeny of human information processing. In the following, we will present one such model (e.g., Li \& Lindenberger, 1999), which has been inspired by the neural noise hypothesis of cognitive aging. According to this model, agebased decrements in the modulation of neuronal signals negatively affect the distinctiveness of cortical representations, with widespread consequences for performance level, variance, and covariation. Most important, this model attempts to link microgenesis to ontogeny, univariate variation to global change, and neuronal mechanisms to behavior ( $\mathrm{Li}$, Lindenberger, et al., 2001).

About 40 years ago, Welford (1965) hypothesized that senescing brains are marked by an increase in neural noise. At a more cognitive level of analysis, Craik (1983) noted that older adults encode events in a less distinctive manner than young adults because of reduced attentional resources. Relatedly, Kinsboume and Hicks (1978) proposed the notion of functional cerebral space to better understand attention regulation, interference, and individual differences in performance. Here, the metaphor of declining processing resources was reframed as an aging-induced compression of functional cerebral space. In all three cases, behavioral aging was linked to some form of representational dedifferentiation, or a decrease in the distinctiveness of brain states, well in line with the differentiation/dedifferentiation hypothesis pursued independently in psychometrics and foreshadowing the results of current-day brain-imaging studies.

Still another line of recent research has linked behavioral aging to continuous age-associated shifts in neurochemical functioning. Specifically, the dopaminergic system has been identified as a promising neurochemical correlate of behavioral aging (Bäckman \& Farde, 2004). First, dopamine transmitter content and binding mechanisms show an almost universal and highly consistent pattern of age-associated decline in various brain regions during normal aging. Second, much of behavioral aging has been attributed to prefrontal cortex dysfunction (Raz, 2000), where dopam- inergic pathways serve to activate and maintain representations in the absence of environmental cues, and to direct attention to goal-relevant stimuli, actions, and action effects (Miller \& Cohen, 2001). Third, more direct experimental evidence based on animal models points to the functional significance of dopamine receptor density. Taken together, age-associated differences in dopaminergic neuromodulation appear to be related to negative age differences in behavior.

The theory of representational dedifferentiation proposed by S.C. Li and colleagues simulates the effects of aging-related changes in neuromodulation on behavior through a series of neurocomputational simulations. It combines earlier theoretical propositions regarding representational dedifferentiation with recent neurochemical evidence about adult age differences in neuromodulation. Variations in the gain parameter $(G)$ of connectionist models are used to simulate the effects of age differences in neuromodulation on neural network signal processing and behavior. $G$ regulates the imput sensitivity of the network's processing units. When $G$ is at its lower boundary, units become completely insensitive to input variations. With high values of $G$, processing units produce very little output with signals below a certain threshold, and close to maximum output for signals above threshold (see Figure 21.3, top panel). Thus, reductions in $G$ flatten the sigmoidal activation function, thereby reducing a unit's capacity to discriminate between different levels of input signals.

In all neurocomputational simulations used to implement the theory, the only difference between "young" and "old" networks concerns mean levels of $G$; in all other respects, the two groups of networks are identical. In contrast to earlier work (ServanSchreiber, Printz, \& Cohen, 1990), empirical evidence about natural fluctuations in transmitter availability is considered by randomly sampling $G$ from a given distribution at each processing step. Thus, the difference between "old" and "young" networks concerns the mean of the range from which $G$ is sampled; for instance, the Gs of "young" networks are sampled from a range of 0.6 to 1.0 , whereas the Gs of "old" networks are sampled from a range of 0.1 to 0.5 (see Figure 21.3, top panel). To reiterate, both the range and the distribution of the gain parameters and all other aspects of the network do not differ between "young" and "old" networks; the only difference by design concerns the mean level at which the gain parameter is varied. 
'I he most basic consequence of the difference in stochastic gain between "young" and "old" networks is seen in the middle panel of Figure 21.3. This figure displays the output activation of two units with high versus low mean gain in response to the same signal. In the unit with the lower mean gain, output activation is less extreme and more variable. The units' increase in stochastic output variability fundamentally alters the network's state space. As illustrated in the bottom panel of Figure 21.3, networks with a greater proportion of stochastic output variation to a given signal are less capable of mapping different patterns of input signals onto discriminable activation patterns, or network states. 'l'he panel displays the internal activation patterns across five different units to four different stimuli in an episodic memory task. The activation patterns, or internal representations, of the four stimuli are much less discriminable in the "old" than in the "young" network

Decrements in stochastic gain have been used to simulate aging phenomena at both neural and behavioral levels of analysis. Table 21.4 provides a listing of phenomena simulated thus far. As noted already, decrements in gain lead to increments in stochastic variability and to decrements in the distinctiveness of activation patterns. In addition, decrements in gain also can account for less distinct cortical representations (Li, Lindenberger, et al., 2001; Li, Naveh-Benjamin, \& Lindenberger, 2005) and neural-network dedifferentiation at the level of processing modules ( $\mathrm{L}_{i}$ $\&$ Sikström, 2002). Thus, at the neural simulation level, the gain manipulation successfully simulates the "contraction of functional cerebral space" envisioned by Kinsbourne and Hicks (1978) and observed by Park et al. (2004) in the domain of visual perception.

At the behavioral level, comparisons between groups of high-gain networks (young networks) and groups of low-gain networks (old networks) reveal striking similarities to comparisons between groups of young and old individuals (see Figure 21.4). First, mean level of performance is lower among old networks than among young networks. Second, inter-individual differences are larger among old networks than among young networks. Third, intercorrelations between tasks are higher among old networks than among young networks. Figure 21.4 illustrates all of these findings with respect to two different word lists leamed by 20 "young", 20 "middleaged," and 20 "old networks" (Li \& I, indenberger, 1999; Li, Lindenberger, \& Frensch, 2000).

TABLE 21.4. Relating neuromodulation to cognitive aging by reducing the mean of distribution of the gain parameter $(G)$ of neural networks: List of simulated phenomena at neural and behavioral levels of analysis

\section{Neural Aging}

- Increase in stochastic, stimulus-unrelated fluctuation of neuronal output activation (reduced processing robustness)

- Less distinct internal representations (e.g., dedifferentiation of neural activation patterns)

- I.ess distinct processing pathways (e.g., dedifferentiation of functional connectivity)

Behavioral Aging

- Increase in performance fluctuation (within-network variability)

- Decrease in mean levels of performance (e.g., list learning, categony discrimination)

- Increase in interindividual differences in performance

- Increase in covariation between tasks across individuals

- Greater age deficits with more difficult tasks (e.g., ordinal interactions between task difficulty and age in 5-item vs. 8-iten list lcarning)

- Greater proactive interference (e.g., greater age differences in A-B, A-C relative to A-B, C-D list learning)

- Lower maximum levels of performance (e.g., age differences in asymptotes of list learning performance)

- Conjunctive binding deficit in associative learning (e.g., simulating empirical results reported by Naveh-Benjamin $(2000)^{b}$

Note: Reductions in the gain parameter $G$ of neural networks flatten the sigmoidal shape of a unit's activation function, thereby leading to decrements in the total amount as well as increments in the stochastic, stimulus-unrelated componcut of output activation (see Figure 21 . 3, top pancl). (Unless noted otherwise, the list refers to simulations reported in Li, 2002; Li and Lindenberger, (1999); Li, Lindenberger, \& Frensch, (2000); and Li, Lindenberger, and Sikström, 2001. Phenomena printed in italics are discussed in more detail in the present chalpter.)

"Based on Li \& Sikström (2002).

hBased on Li \& Linclenberger (in press). 

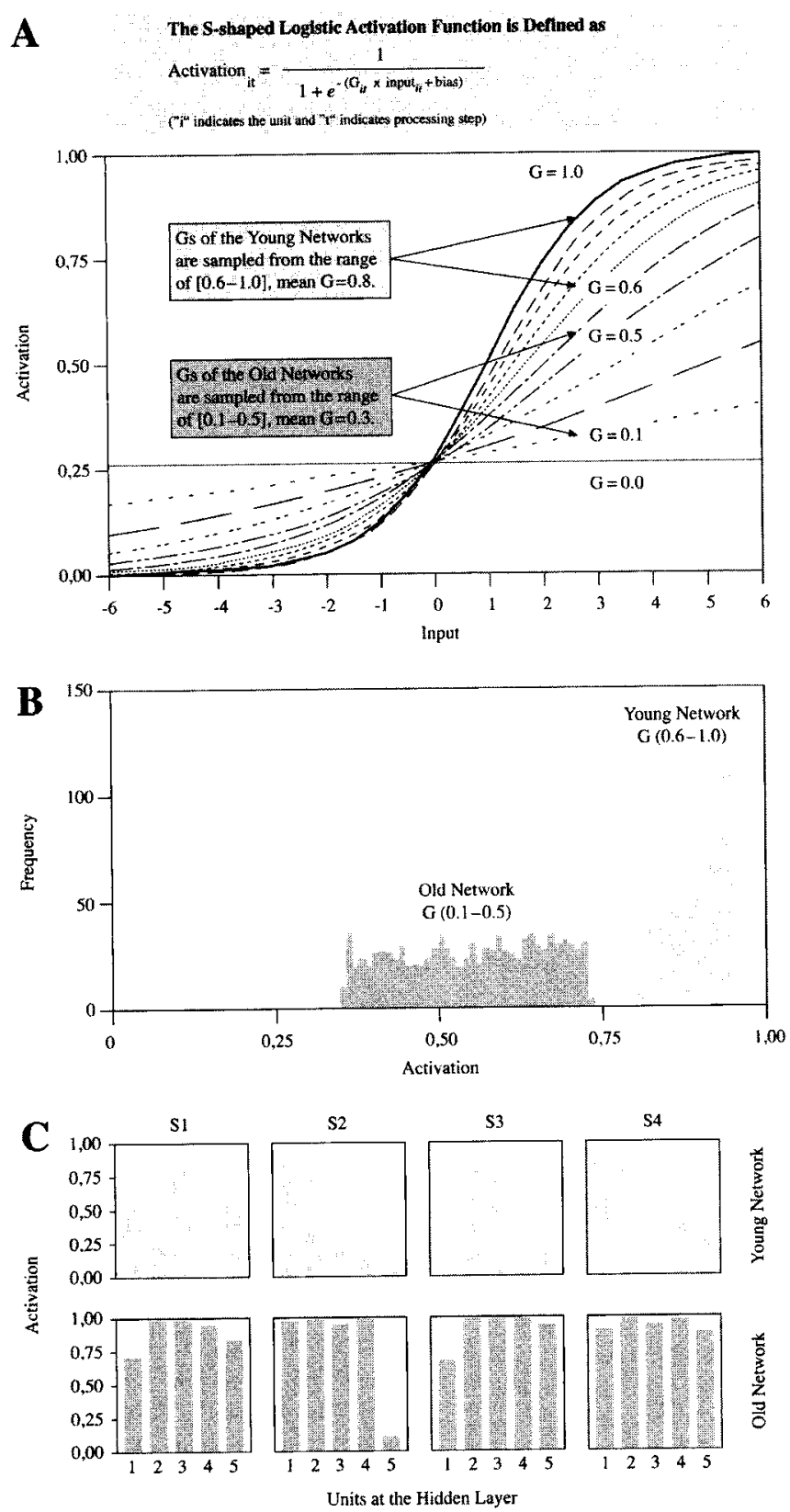

FIGURE 21.3. (A) The S-shaped logistic activation function at different values of the gain parameter $(G)$. Physiological evidence suggests that a logistic function with a negative bias captures the function relating the strength of an input signal to a neuron's firing rate, with the slope being steepest around baseline. Reducing mean $G$ flattens the activation function so that a unit becomes less responsive. (B) $G$ and the variability of activation across processing steps. Reducing mean $G$ increases the temporal variability of a unit's response to an identical imput signal. (C) Activation patterns across five hidden units of one "young" and one "old" network in response to four different stimuli $\left(\mathrm{S}_{1}\right.$ to $\mathrm{S}_{4}$ ). The internal representations of the four stimuli are much less differentiable in the "old" than in the "young" network. (Panels $(A)$ to $(C)$ are adapted from Li et al., 2000.) 

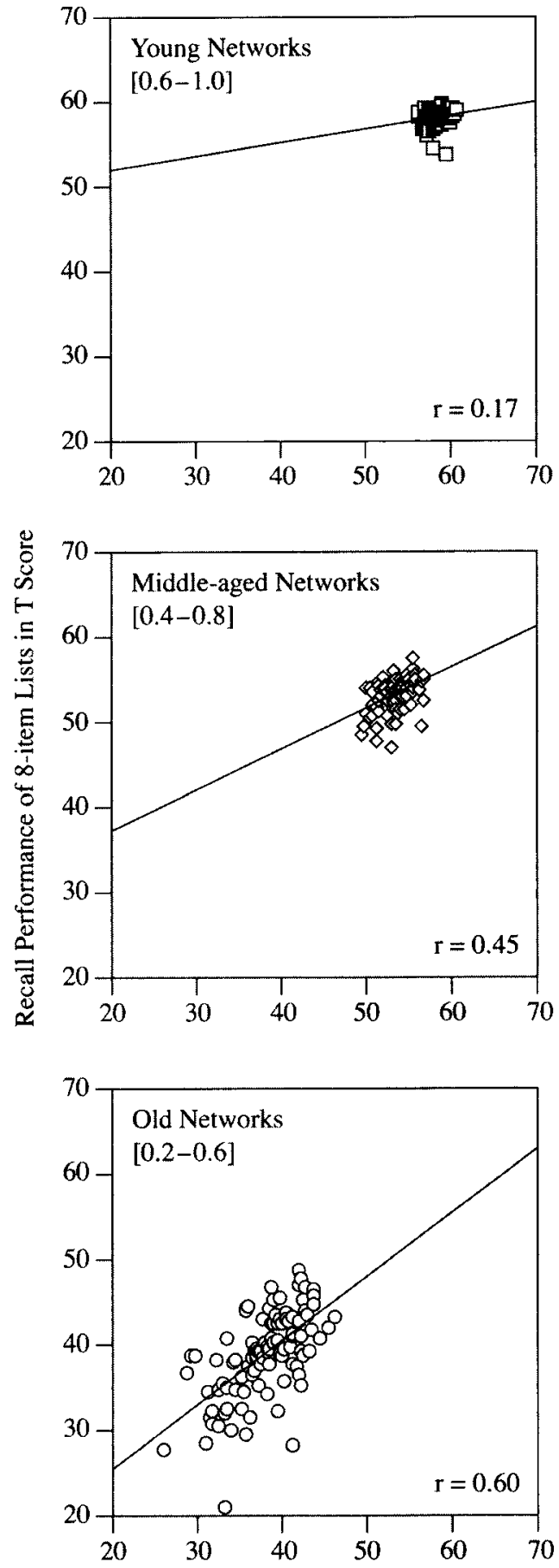

Recall Performance of 3-item Lists in T Score
To conclude, these simulations show that increments in stochastic (i.e., stimulus-unrelated) variability induced by lowering the gain parameter of connectionist networks may lead to decrements in means as well as increments in variance and correlations at the population level. Age differences in the structure of inter-individual differences were solely brought about by increased stochastic variability at the intra-individual (i.e., intra-network) level (for more details, e.g., Li \& Lindenberger, 1999); no additional assumptions about differences in life histories or antecedent conditions were needed. Hence, the present model specifies reductions in the mean of stochastic gain as a mechanism that links univariate microgenetic variability to ontogenetic changes in functional organization, tracing a path from the upper left to the lower right cell of the taxonomy presented in Table 21.1.

\section{OUTLOOK}

Interest in variability as an agent of development during adulthood and old age has increased during the last decade. Researchers increasingly recognize the need to assess and articulate various forms and functions of variability, and to identify relevant mechanisms (Hultsch \& MacDonald, 2004; Lövdén \& Lindenberger, 2005; Rabbitt, Osman, Moore, \& Stollery, 2001). General theories of cognitive aging that emphasize central tendency such as average response latency (e.g., Salthouse, 1996) have been augmented by formal theories that emphasize stochastic variability at various levels of analysis (e.g., Li \& Lindenberger, 1999; Ratcliff et al., 2000). For instance, recent empirical findings (e.g., Li, Lindenberger, et al., 2004; Nesselroade \& Salthouse, 2004) suggest that inter-individual differences in processing fluctuation may functions as traits that change with age and predict mean levels of performance.

FIGURE 21.4. Scatterplots of between-network (i.e., inter-individual) correlations between memory performance for a 3-item list and memory performance for an 8-item list in groups of networks with high, medium, and low mean values of stochastic gain $(G)$. (Modified after Li and Lindenberger, 1999.) 
We agree with Nesselroade and Salthouse (2004) that classical test-theory interpretations of observed between-person variability need to be abandoned once and for all (cf. Hertzog, 1985). The contributions of intra-person fluctuations to observed between-person variability are simply too large and too systematic to be accommodated by a theory that makes no principled reference to variability at the within-person level. Researchers' increasing reliance on statistical procedures capable of representing inter-individual differences in intra-individual change (e.g., Baltes et al., 1977/1988) indicates a change for the better (e.g., Lindenberger \& Ghisletta, 2004; Wilson et al., 2002)

There continues to be a dearth of data on age differences in various forms of microgenetic variabilitytrial-by-trial, session-to-session, and day-to-day (but see Li, Aggen, et al., 2001; MacDonald et al., 2003; Nesselroade \& Salthouse, 2004; Rabbitt et al., 2001). Extensive multivariate time series of carefully selected collections of individuals, young and old, are needed to investigate the functional status and multiple meanings of variability (e.g., Jones \& Nesselroade, 1990). Ideally, behavioral and neural variables should be assessed conjointly to examine links between variability at both levels of analysis. To provide more direct tests of the differentiation/dedifferentiation hypothesis, intra-person structures of intellectual abilities need to be assessed at different ages and stages of learning. The ensuing comparisons between intra-person and interperson structures will help to quantify sample heterogeneity and departure from variation equivalence. The phenomenon of strategic diversity and other forms of adaptive microgenetic variability also awaits further study, including the difficult issue of how different strategies emerge in the first place (e.g., Lautrey, 2003; chapter 20 , this volume). The selection, optimization, and compensation (SOC) model of successful development offers a productive theoretical context for examining this issue (e.g., Baltes \& Baltes, 1990; Krampe \& Baltes, 2003).

Finally, the role of noise in aging systems needs further exploration. Generally, noise is assumed to degrade information processing in physical and biological systems. However, under certain conditions, noise actually enhances rather than hinders the detection of weak signals. The simplest possible system showing this property consists of a threshold, a subthreshold signal, and added noise. Whenever the noise plus the signal crosses the threshold in one direction, it triggers a response in the output. As a detector of weak signals, this system is optimally sensitive at some non-zero level of input noise. Recently, researchers have begun to examine this phenomenon, called stochastic resonance (Wiesenfeld \& Moss, 1995), in aging individuals (e.g., Collins et al., 2003). Future work needs to examine the fate of stochastic resonance in networks with low stochastic gain in order to specify optimal levels of external noise for signal processing in old age (Li, Oertzen, \& Lindenberger, in press).

ACKNOWLedgments We thank Paul Baltes, Ellen Bialystok, and Gus Craik for comments on an earlier version of the present chapter, and Shu-Chen Li for many helpful and stimulating discussions.

\section{References}

Allaire, J. C., \& Marsiske, M. (2002, April). Intra-individual variability in cognitive functioning: Is inconsistency an index of vulnerability? Paper presented at the Cognitive Aging Conference, Atlanta, Georgia.

Bäckman, L., \& Farde, L. (2004). The role of dopamine systems in cognitive aging. In R. Cabeza, L. Nyberg. \& D. C. Park (Eds.), Cognitive neuroscience of aging: Linking cognitive and cerebral aging (pp. 5884). New York: Oxford University Press.

Baltes, P. B. (1968). Longtitudinal and cross-sectional sequences in the study of age and generation effects. Human Development, 11, 145-171.

Baltes, P. B. (1987). Theoretical propositions of life-span developmental psychology: On the dynamics between growth and decline. Developmental Psychology, 23, 611-626.

Baltes, P. B., \& Baltes, M. M. (1990). Psychological perspectives on successful aging: The model of selective optimization with compensation. In P. B. Baltes $\&$ M. M. Baltes (E.ds.), Succcessful aging: Perspectives from the behavioral sciences (pp. 1-34). New York: Cambridge University Press.

Baltes, P. B., Comelius, S. W., Spiro, A., Nesselroade, J. R., \& Willis, S. L. (1980). Integration vs. differentiation of fluid-crystallized intelligence in old age. Developmental Psychology, 16, 625-635.

Baltes, P. B., \& Lindenberger, U. (1997). Emergence of a powerful connection between sensory and cognitive functions across the adult life span: A new window at the study of cognitive aging? Psychology and Aging, 12, 12-21

Baltes, P. B., Lindenberger, U., \& Staudinger, U. M. (1998). Life-span theory in developmental psychology. In R. M. Lerner (Ed.), Handbook of child psychology: Vol. 1. Theoretical models of human 
development (5th ed., pp. 1029-1143). New York: Wiley.

Baltes, P. B., Reese, H. W., \& Nesselroade, J. R. (1977/ 1988). Life-span developmental psychology: Introduction to research methods. Hillsdale, NJ Lawrence Erlbaum Associates. Buss, A. R. (1974). A general developmental model for interindividual differences, intraindividual differences, and intraindividual changes. Developmental Psychology, 10, $70-78$.

Cabeza, R. (2002). Hemispheric asymmetry reduction in older adults: 'The HAROLD model. Psychology and Aging, 17, 85-100.

Cattell, R. B. (1966). The data box: Its ordering of total resources in terms of possible relational systems. In R. B. Cattell (Ed.), Handbook of multivariate experimental psychology (pp. 67-128). Chicago: Rand McNally

Cattell, R. B. (1971). Abilities: Their structure, growth, and action. Boston, MA: Houghton Mifflin.

Collins, J. J., Priplata, A. A., Gravelle, D. C., Niemi, J., Harry, J., \& Lipsitz, L. A. (2003). Noise-enhanced human sensorimotor function. IEEE Engineering in Medicine and Biology Magazine, March/April, $76-83$.

Craik, F. I. M. (1983). On the transfer of information from temporary to permanent memory. Philosophical Transactions of the Royal Society of London, B302, 341-359.

Deary, I. J., Egan, V., Gibson, G. J., Austin, E. J., Brand, C. R., \& Kellaghan, T. (1996). Intelligence and the differentiation hypothesis. Intelligence, 23, 105-132.

Esstes, W. K. (1956). The problem of inference from curves based on group data. Psychological Bulletin, $53,134-140$

Garrett, H. E. (1946). A developmental theory of intelligence. American Psychologist, 1, 372-378.

Hertzog, C. (1985). An individual differences perspective: Implications for cognitive research in gerontology. Research on Aging, 7, 7-45.

Hofer, S. M., \& Sliwinski, M. J. (2001). Understanding aging: An evaluation of research designs for assessing the interdependence of ageing-related changes. Gerontology, 47, 341-352.

Hom, J. L. (1989). Models of intelligence. In R. L. Linn (Ed.), Intelligence: Measurement, theory, and public policy (pp. 29-73). Urbana, IL: University of Iljinois Press.

Hultsch, D. F., Hertzog, C., Dixon, R. A., \& Small, B. J. (1998). Memory change in the aged. Cambridge, UK: Cambridge University Press.

Hultsch, D. F., \& MacDonald, S. W. S. (2004). Intraindividual variability in performance as a theoretical window onto cognitive aging. In R. A. Dixon,
L. Bäckman \& L.-G. Nilsson (Eds.), New frontiers in cognitive aging (pp. 65-88). New York: Oxford University Press.

Jones, C. J., \& Nesselroade, J. R. (1990). Multivariate, replicated, single-subject, repeated measures designs and P-technique factor analysis: A review of intraindividual change studies. Experimental Aging Research, 16, 171-183

Kinsbourne, M., \& Hicks, R. E. (1978). Functional cerebral space: A model for overflow, transfer and interference effects in human performance: A tutorial review. In J. Requin (Ed.), Attention and performance VII, (PP. 345-362). Hillsdale, NJ: Lawrence Erlbaum Associates.

Krampe, R. T., \& Baltes, P. B. (2003). Intelligence as adaptive resource development and resource allocation: A new look through the lenses of SOC and expertise. In R. J. Stemberg \& E. L. Grigorenko (E.ds.), Perspectives on the psychology of abilities, competencies, and expertise (pp. 31-69). New York: Cambridge University Press.

Lautrey, J. (2003). A pluralistic approach to cognitive differentiation and development. In R. J. Stemberg, J. Lautrey \& T. Lubart (Eds.), Models of intelligence: International perspectives (pp. 117-131). Washington, DC: American Psychological Association.

Li, K. Z. H., \& Lindenberger, U. (2002). Relations between aging sensory/sensorimotor and cognitive functions. Neuroscience and Biobehavioral Reviews, 26, 777-783.

Li, S.-C. (2002). Connecting the many levels and facets of cognitive aging. Current Directions in Psychological Science, 11, 38-43.

Li, S.-C., Aggen, S., Nesselroade, J. R., \& Baltes, P. B. (2001). Short-term fluctuations in elderly people's sensorimotor functioning predict text and spatial memory performance. Gerontology, 47, 100-116.

Li, S.-C., Huxhold, O., \& Schmiedek, F. (2004). Aging and attenuated processing robustness. Gerontology, $50,28-34$.

Li, S.-C., Lindenberger, L., \& Sikström, S. (2001). Aging cognition: From neuromodulation to representation. Trends in Cognitive Science, 5, 479-486.

Li, S.-C., \& Lindenberger, U. (1999). Cross-level unification: A computational exploration of the link between deterioration of neurotransmitter systems and dedifferentiation of cognitive abilities in old age. In L.-G. Nilsson \& H. Markowitsch (Eds.), Cognitive neuroscience of memory (pp. 104-146). Toronto: Hogrefe \& Huber.

Li, S.-C., \& Lindenberger, U. (in press). Aging deficits in neuromodulation of representational distinctiveness and conjunctive binding: Computational explorations of possible links. In H. D. Zimmer, A. 
Mecklinger \& U. Lindenberger (Eds.), Binding in human memon: A neurocognitive approach. New York: Oxford University Press.

L,i, S.C., Lindenberger, U., \& Frensch, P. A. (2000). Unifying cognitive aging: From neuromodulation to representation to cognition. Neurocomputing. $32-33,879-890$.

Li, S.-C., Lindenberger, U., Hommel, B., Aschersleben, G., Prinz, W., \& Baltes, P. B. (2004). Lifespan transformations in the couplings among intellectual abilities and constituent cognitive processes. Psychological Science, 15, 155-163.

Li, S.-C., Naveh-Benjamin, M., \& Lindenberger, U. (2005). Aging neuromodulation impairs associative binding: A neurocomputational account. Psychological Science, 16, 445-450.

I,i, S.-C., Oertzen, T. V., \& Lindenberger, U. (in press). A neurocomputational model of stochastic resonance and aging. Neurocomputing.

Li, S.-C., \& Sikström, S. (2002). Integrative neurocomputational perspectives on cognitive aging, neuromodulation, and representation. Neuroscience and Biobehavioral Reviews, 26, 795-808.

Lindenberger, U. (2001). Lifespan theories of cognitive development. In N. J. Smelser \& P. B. Baltes (Eds.), International encyclopedia of the social o behavioral sciences (Vol. 13, pp. 8848-8854). Amsterdam: Elsevier Science.

Lindenberger, U., \& Baltes, P. B. (1994). Aging and intelligence. In R. J. Stemberg (Ed.), Encyclopedia of human intelligence (Vol. 1, pp. 52-66). New York: Macmillan.

Lindenberger, U., \& Baltes, P. B. (1995). Testing-thelimits and experimental simulation: Two methods to explicate the role of learning in development. $\mathrm{Hu}$ man Development, 38, 349-360.

Lindenberger, U., \& Baltes, P. B. (1997). Intellectual functioning in old and very old age: Cross-sectional results from the Berlin Aging Study. Psychology and Aging, 12, 410-432.

Lindenberger, U., \& Baltes, P. B. (1999). Die Entwicklungspsychologie der Lebensspanne (LifespanPsychologie): Johann Nicolaus Tetens zu Ehren (1736-1807). Zeitschrift für Psychologie, 207, 299323.

Lindenberger , U., \& Ghisletta, P. (2004). Modeling longitudinal changes in old age: From covariance structures to dynamic systems. In R. A. Dixon, L. Bäckman \& L.-G. Nilsson (Eds.), New frontiers in cognitive aging (pp. 199-216). New York: Oxford University Press.

Lindenberger, U., Singer, 'T., \& Baltes, P. B. (2002). Longitudinal selectivity in aging populations: Separating mortality-associated versus experimental components in the Berlin Aging Study (BASE). Journal of Gerontology: Psychological Sciences, 57B(6), 474-482.

Lövdén, M., Ghisletta, P., \& Lindenberger, U. (2004) Cognition in the Berlin Aging Study (BASE): The first ten years. Aging, Neuropsychology, and Cognition, 11, 104-133..

Lövdén, M., \& Lindenberger, U. (2005). Development of intellectual abilities in old age: From age gradients to individuals. In $\mathrm{O}$. Wilhelm \& R. W. Fingle (Eds.), Understanding and measuring intelligence (pp. 203-221). Thousand Oaks, CA: Sage.

MacDonald, S. W. S., Hultsch, D. F., \& Dixon, R. A. (2003). Performance variability is related to change in cognition: Evidence from the Victoria Longitudinal Study. Psychology and Aging, 18, 510-523.

Magnusson, D., \& Stattin, H. (1998). Person-context interaction theories. In R. M. Lerner (F.d.), Theoretical models of human development. Volume 1 : Handbook of child psychology (5th ed.) (pp. 685759). New York: Wiley.

Miller, E. K., \& Cohen, J. D. (2001). An integrative theory of prefontal cortex function. Annual Review of Neuroscience, 24, 167-202.

Molenaar, P. C. M., Huizenga, H. M., \& Nesselroade, I. R. (2003). The relationship between the structure of inter-individual and intra-individual variability: A theoretical and empirical vindication of developmental systems theory. In U. M. Staudinger \& U. Lindenberger (Eds.), Understanding human development: Lifespan psychology in exchange with other disciplines (pp. 339-360). Dordrecht, Netherlands: Kluwer Academic Publishers.

Nesselroade, J. R. (1991). The warp and the woof of the developmental fabric. In R. M. Downs, L. S. Liben $\&$ D. S. Palermo (Eds.), Visions of aesthetics, the environment and development: The legacy of Joachim Wohlwill (pp. 213-240). Hillsdale, NJ: Lawrence Erlbaum Associates.

Nesselroade, J. R., \& Salthouse, T. A. (2004). Methodological and theoretical implications of intraindividual variability in perceptual motor performance. Joumal of Gerontology: Psychological Sciences, 59B, P49-P55.

Nesselroade, J. R., \& Thompson, W. W. (1995). Selection and related threats to group comparisions: An example comparing factorial structures of higher and lower ability groups of adult twins. Psychological Bulletin, 117, 271-284.

Park, D. C., Lautenschlager, G., Hedden, T., Davidson, N. S., Smith, A. D., \& Smith, P. K. (2002). Models of visuospatial and verbal memory across the adult lifespan. Psychology and Aging, 17, 299-320.

Park, D. C., Polk, T. A., Park, R., Minear, M., Savage, 
A., \& Sinith, M. R. (2004). Aging reduces neural specialization in ventral visual cortex. PNAS, 101, 13091-13095.

Rabbitt, P., Osman, P., Moore, B., \& Stollery, B. (2001) There are stable individual differences in performance variability, both from moment to moment and from day to day. The Quarterly Journal of Experimental Psychology, 54A, 981-1003.

Ratcliff, R., Spieler, D. H., \& McKoon, G. (2000). Explicitly modeling the effects of aging on response time. Psychonomic Bulletin and Review, 7, 1-25.

Raz, N. (2000). Aging of the brain and its impact on cognitive performance: Integration of structural and functional findings. In F. I. M. Craik \& T. A. Salthouse (Eds.), The handbook of aging and cognition (2 ed., pp. 1-90). Mahwah, NJ: Lawrence Erlbaum Associates.

Reinert, G. (1970). Comparative factor analytic studies of intelligence throughout the human life span. In L. R. Goulet \& P. B. Baltes (Eds.), Life-span developmental psychology: Research and theory (pp. 476484). New York: Academic Press.

Reuchlin, M. (1978). Processus vicariants et différences individuelles. Joumal de Psychologie, 2, 133-145.

Salthouse, T. A. (1991). Theoretical perspectives on cognitive aging. Hillsdale, NJ: Lawrence Erlbaum Associates.

Salthouse, T. A. (1996). The processing-speed theory of adult age differences in cognition. Psychological Review, 103, 403-428.

Schaie, K. W., Maitland, S. B., Willis, S. L., \& Intieri, R. C. (1998). Longitudinal invariance of adult psychometric ability factor structures across 7 years. Psychology and Aging, 13, 8-20.

Schreurs, B. G., Bahro, M., Molchan, S. E., Sunderland, T., \& McIntosh, A. R. (2001). Interactions of prefrontal cortex during eyeblink conditioning as a function of age. Neurobiology of Aging, 22, 237-246.
Servan-Schreiber, D., Printz, H., \& Cohen, J. D. (1990). A network model of catecholamine effects: Gain, signal-to-noise ratio, and behavior. Science, 249, 892-894.

Siegler, R. S. (1989). Mechanisms of cognitive development. Annual Review of Psychology, 40, 353-379.

Singer, T., Lindenberger, U., \& Baltes, P. B. (2003). Plasticity of memory for new learning in very old age: A story of major loss? Psychology and Aging, 18, 306-317.

Singer, T., Verhaeghen, P., Ghisletta, P., Lindenberger, U., \& Baltes, P. B. (2003). The fate of cognition in very old age: Six-year longitudinal findings in the Berlin Aging Study. Psychology and Aging, 18, 318-331.

Smith, L. B., \& Thelen, E. (2003). Development as a dynamic system. Trends in Cognitive Science, 7 , 343-348.

Tetens, J. N. (1777). Philosophische Versuche über die menschliche Natur und ihre Entwicklung. Leipzig, Germany: Weidmanns Erben und Reich.

Thaler, D. S. (2002). Design of an aging brain. Neurobiology of Aging, 23, 13-15.

Welford, A. 'T. (1965). Perfomance, biological mechanisms, and age: A theoretical sketch. In A. T. Welford \& J. E. Birren (Eds.), Behavior, aging, and the nervous system (pp. 3-20). Springfield, IL: Thomas.

Wiesenfeld, K., \& Moss, F. (1995). Stochastic resonance and the benefits of noise: From ice ages to crayfish and SQUIDs. Nature, 373, 33-36.

Wilson, R. S., Beckett, L. A., Barnes, L. L., Schneider, J. A., Bach, J., Evans, D. A., \& Bennett, D. A. (2002). Individual differences in rates of change in cognitive abilities in older persons. Psychology \& Aging, 17, 179-193.

Wohlwill, J. F. (1973). The study of behavioral development. New York: Academic Press. 


\title{
LIFESPAN COGNITION MECHANISMS OF CHANGE
}

\author{
EDITED BY
}

Ellen Bialystok

Fergus I. M. Craik

\section{OXFORD \\ UNIVERSITY PRESS

